\title{
Article
}

\section{Post-COVID-19 Condition and Health Status}

\author{
Antarpreet Kaur ${ }^{1}$, Chloe Michalopoulos ${ }^{2} \mathbb{D}$, Suzanne Carpe ${ }^{2}$, Soontharee Congrete ${ }^{1}\left(\mathbb{D}\right.$, Hira Shahzad $^{1}$, \\ Jane Reardon ${ }^{1}$, Dorothy Wakefield ${ }^{1}$ (D) , Charles Swart ${ }^{3}$ and Richard ZuWallack ${ }^{1, *}$
}

1 Pulmonary and Critical Care, St Francis Hospital-Trinity Health of New England, Hartford, CT 06105, USA; antarpreet.kaur@trinityhealthofne.org (A.K.); congrete@uchc.edu (S.C.); hira.shahzad001@trinityhealthofne.org (H.S.); jane.reardon@trinityhealthofne.org (J.R.); jewakefield@snet.net (D.W.)

2 Department of Biology, Trinity College, Hartford, CT 06106, USA; chloe.michalopoulos@trincoll.edu (C.M.); suzanne.carpe@trincoll.edu (S.C.)

3 Neuroscience Program, Trinity College, Hartford, CT 06106, USA; charles.swart@trincoll.edu

* Correspondence: rlzuwallack@gmail.com; Tel.: +860-714-4055; Fax: 860-714-8035

check for updates

Citation: Kaur, A.; Michalopoulos, C.; Carpe, S.; Congrete, S.; Shahzad, H.; Reardon, J.; Wakefield, D.; Swart, C.; ZuWallack, R. Post-COVID-19 Condition and Health Status. COVID 2022, 2, 76-86. https://doi.org/ $10.3390 /$ covid2010006

Academic Editor: Dora Marinova

Received: 23 December 2021

Accepted: 11 January 2022

Published: 14 January 2022

Publisher's Note: MDPI stays neutral with regard to jurisdictional claims in published maps and institutional affiliations.

Copyright: (C) 2022 by the authors. Licensee MDPI, Basel, Switzerland. This article is an open access article distributed under the terms and conditions of the Creative Commons Attribution (CC BY) license (https:// creativecommons.org/licenses/by/ $4.0 /)$.

\begin{abstract}
Background: Observational studies of the long-term effects of COVID-19 infection generally focus on individual symptoms rather than health status. Objective: Longitudinal assessment of general health status following COVID-19 infection. Design: Observational study, with data collected from two telephone surveys at $32 \pm 10$ and $89 \pm 25$ days after discharge from the hospital or emergency department (ED) for a COVID-19 infection. Medicaid or no insurance was our marker of low socioeconomic status (SES). Acute disease severity was determined by summing 10 severity markers (yes-no) from the health encounter. Baseline comorbidity was a modified Charlson Index. Participants: 40 patients. Mean age was $54 \pm 15$ years, 50\% were female, and $40 \%$ had low socioeconomic status. Main Measures: (1) the 20-item Medical Outcomes Study Short-Form General Health Survey (SF-20); (2) Dyspnea (modified Medical Research Council); (3) Psychological symptoms (Patient Health Questionnaire for Anxiety and Depression); (4) Cognitive function (Cognitive Change Questionnaire); (5) Fatigue (Short Fatigue Questionnaire); (6) A 10-item review of systems (ROS) questionnaire. Key Results: Percentages with abnormal symptoms at the first and second surveys were (respectively): Dyspnea (40,33), Fatigue $(53,50)$, Anxiety $(33,18)$, Depression $(20,10)$, PHQ-4 Composite $(25,13)$, and Cognitive $(18,10)$. Mean scores on the SF-20 subscales, Physical Functioning, Role Functioning, Social Functioning, Health Perception, Mental Health, and Pain were numerically lower than means from a published study of elderly outpatients. With the exception of Pain, all SF-20 subscale scores improved significantly by the second survey. In multivariable analyses, dyspnea was predictive of impairment in all SF-20 subscales at the second survey. Conclusions: COVID-19 infection causes persistent abnormality across multiple patient-reported outcome areas, including health status. The persistence of impairment in each health status component is influenced by baseline dyspnea.
\end{abstract}

Keywords: COVID-19; health status; health-related quality of life; dyspnea; long-haul COVID; Post-COVID-19 Condition

\section{Introduction}

Acute COVID-19 disease is commonly complicated by prolonged symptoms that can often last for months [1,2]. These symptoms can include fatigue, dyspnea, cognitive and mental impairments, chest pains, joint pains, palpitations, myalgias, alterations or loss of taste or smell, cough, headache, and gastrointestinal distress [3]. With the recognition of these post-infection sequelae by the medical and lay community, the terms long COVID or long-haul COVID have been commonly used. Recently, a Delphi process conducted by an international panel of patients, clinicians, and researchers under the auspices of the World Health Organization arrived at a consensus terminology and definition for this condition: "Post-COVID-19 condition occurs in individuals with a history of probable or confirmed 
SARS-CoV-2 infection, usually 3 months from the onset, with symptoms that last for at least 2 months and cannot be explained by an alternative diagnosis. [4]".

While the focus of investigation on Post-COVID-19 Condition has been on persistent symptoms, there is a relative paucity of research on impairments in general health status. Since health status assessment provides a deeper, personal perspective of the pervasive effects of the disease, its comorbidity, and its treatments [5,6], clinical research in this area might provide additional perspective in assessing the impact of COVID-19 on the patient. Accordingly, we assessed the impact of COVID-19 on health status, testing its trajectory and the clinical factors that may influence it.

\section{Methods}

This observational study was approved by our Institutional Review Board (Trinity Health of New England Institutional Review Board, Assurance \#FWA00020300, approval 28 September 2021) and informed consent was obtained from all participants. Our primary aim was to evaluate longitudinal impairments in health status following COVID-19 infection. Our secondary aim was to evaluate factors related to health status impairment.

Inclusion criteria were: (1) Adults discharged from our hospital or its emergency department (ED) to home after treatment for COVID-19; (2) Confirmation of COVID-19 infection by laboratory testing; and (3) Consent to participate at the initial and subsequent telephone encounters. Exclusion criteria were: (1) A medical event that led to the index health care encounter other than the COVID-19 infection; and (2) Cognitive issues or a perceived language barrier (determined from the initial record review) that would preclude informed consent or adequate participation in the surveys.

We chose survey questionnaires to be brief and amenable to a telephone survey format. These included:

1. The modified Medical Research Council (mMRC) dyspnea questionnaire (0-4 scale) [7] We considered an mMRC rating of 3 or 4 to indicate severe dyspnea.

2. The Patient Health Questionnaire for Anxiety and Depression (PHQ-4). This screening instrument has 4 questions, 2 focusing on anxiety and 2 on depression symptoms, plus a composite score [8]. Severity scores of 3 or greater in either anxiety or depression subscales or a PHQ- 4 composite score $\geq 6$ were considered elevated.

3. The Cognitive Change Questionnaire (CC8) [9] is a screening tool with 8 questions rating cognitive impairment. A score $\geq 2$ was used as a marker for cognitive impairment.

4. The Short Fatigue Questionnaire (SFQ) [10] rates fatigue using 4 questions, each rated on a 1-7-point scale. This is a visual scale with extremes and middle anchored by descriptors. Scores $\geq 18$ were used to represent high levels of fatigue.

5. The Medical Outcomes Study Short-Form General Health Survey (SF-20) [11]. This health status instrument has 20 questions summarized in 6 subscales: physical functioning, role functioning, social functioning, mental health, health perceptions, and pain. Scores are scaled to range from 0 (worst) to 100 (best). Since there are no thresholds indicating abnormality with this instrument, we included for comparison data from the closest cohort of individuals we could find in the medical literature [12].

The telephone survey also included 10 review of systems (ROS) questions, evaluating the presence of cough, excessive sleepiness, chest pain, lower extremity edema, frequent headaches, numbness of feet, arthritis or joint pain, difficulty walking, trouble sleeping and the loss of the sense of taste or smell. Dyspnea, fatigue, and memory assessment was not included in the ROS since they were assessed by questionnaire. If there was a "yes" response to a particular ROS item, the patient was then queried as to whether that symptom was worse or not-worse compared to the time before being infected with COVID-19. These ROS responses $(1=$ yes, $0=$ no) were summed to give a ROS symptom score.

Demographic data abstracted from the electronic record included age, sex, insurance status (Medicaid or no insurance was used as a marker for low socioeconomic status (SES)), and self-identified race and ethnicity. 
Acute COVID-19 clinical severity markers from the record review included: (1) Peak supplemental oxygen requirement $\geq 4 \mathrm{~L} / \mathrm{min}$ (or equivalent); (2) Discharge home with a prescription for supplemental oxygen; (3) Multilobar pneumonia; (4) Intravenous corticosteroid therapy for COVID-19; (5) Remdesivir treatment; (6) Clinical or laboratory cardiac ischemia; (7) Decompensated heart failure; (8) Shock; (9) Use of non-invasive ventilation; and (10) Endotracheal intubation and placement on a respirator. We created an arbitrary severity score by summing the above 10 scores $(0=$ no, $1=$ yes $)$, with the result ranging from 0 (least) to 10 (greatest) acute COVID-19 severity.

Comorbidity was evaluated using a revised and weighted Charlson Comorbidity Index [13]. An earlier version of this was proven predictive of severe COVID-19 outcome [14]. In our analysis the Charlson score was tested as both a continuous variable and after dichotomization to scores above and below 1 . In addition to the Charlson Comorbidity Index variables, we included systemic hypertension, obesity, and asthma as other potentially important comorbid factors predicting outcome.

While the original protocol was to have investigators contact and perform telephone surveys at 1,2, and 3 months, we had unanticipated difficulty in contacting some patients after they initially agreed to participate in the study, especially for the third interview. Because of delays in eventually contacting patients or not reaching some patients at all, the time range for the surveys was broadened and we used the last data available (from either second or third interview) to assess trajectory of outcome data, and added time from discharge to the survey as a covariate. For the purpose of our data presentation the last survey (whether the second or third) was called the second survey.

We used SAS 9.4 for Windows 10 (SAS Institute, Inc., Cary, NC, USA) for statistical analyses, including descriptive analyses. The significance of changes in survey data was determined using paired t-tests. Multivariate stepwise regression (SAS PROC GLMSELECT), (SAS Institute, Inc., Cary, NC, USA) was used to evaluate predictors of each component of health status assessed in each of the two surveys. Independent variables included age, race/ethnicity, obesity (clinical diagnosis), SES, Charlson comorbidity, the composite acute disease severity score, questionnaire-rated dyspnea, psychological variables, fatigue, and cognitive status, and ROS symptom score. In the analyses, the questionnaire variables were dichotomized based on whether they were above or not-above the normal range ( 1 vs. 0$)$ established for the instrument. Charlson Comorbidity Index scores with dichotomized to above and below 1 . Severity scores and ROS summary scores were kept as continuous variables. For those analyses of Survey 2 health status, the corresponding value from survey 1 was added as a covariate to the model and time from the health care discharge to the survey was included as a covariate. A $p<0.05$ was considered significant.

\section{Results}

Figure 1 outlines patient recruitment for the study. Of 96 potentially eligible patients from the record review, 54 provided consent and completed Survey 1 . The causes of noninclusion in Survey 1 included not being reachable by telephone for the survey after having previously provided consent $(n=35)$, withdrawing consent $(n=4)$, and a language barrier identified at the time of the initial contact $(n=3)$. Of the 54 completing survey $1,40(74 \%)$ participated in Survey 2. Reasons for non-participation (dropouts) included not being reachable by telephone $(n=12)$ and withdrawal of consent $(n=2)$. Compared to those who completed the surveys dropouts were younger ( $43 \pm 17$ vs. $53 \pm 15$ years, $p=0.04$ ) and had a lower acute COVID-19 severity score $(2.1 \pm 2.2$ vs. $3.3 \pm 1.8, p=0.04)$. Data from 40 patients completing the two surveys were analyzed. The time between health care discharge and the first and second surveys were $31 \pm 8$ and $89 \pm 25$ days, respectively. 


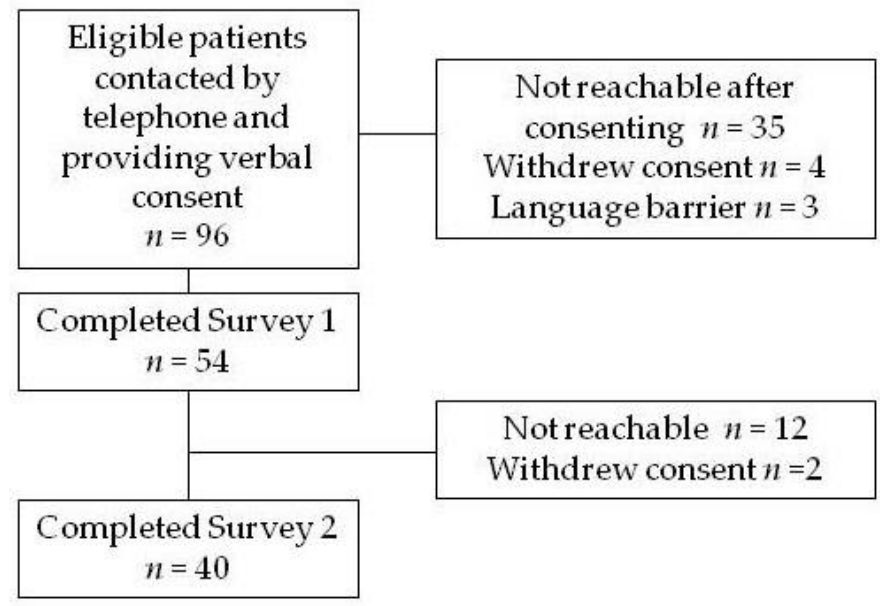

Figure 1. Patient recruitment. See text for description.

Patient characteristics are listed in Table 1, while individual COVID-19 severity variables are listed in Table 2. Age ranged from 25 to 84 , half were female, almost $72 \%$ were non-Hispanic Black or White, and $40 \%$ had low SES. Seventy percent were diagnosed with obesity. Forty percent had at least one comorbid condition; the mean COVID-19 severity score was $3.3 \pm 1.8$. Table 3 lists the percentages of patients with elevated dyspnea, fatigue, and cognitive and psychological scores at the two surveys. Although the percentages with values above the normal range decreased numerically in all outcome areas, none of the changes were significant. Table 4 shows the SF-20 health subscale scores of each survey. Except for the pain score, all subscales showed significant increases (i.e., improvement) at the second survey. In addition, mean subscale scores from a published cohort of Canadians (age range, 66-70 years) living at home [12] is provided in this table for comparison. Although this cohort was considerably older than our sample, it represents the closest age group to ours we could find in the medical literature. Role Functioning, Social Functioning, Mental Health and Health Perceptions appeared considerably lower in our sample, although statistical comparisons could not be performed.

Table 1. Patient characteristics.

\begin{tabular}{cc}
\hline Number of Subjects & 40 \\
Age (years) ( \pm SD) & $53 \pm 8$ \\
Obesity (clinical diagnosis) \% & 70 \\
ED treatment only (\%) & 13 \\
Female (\%) & 50 \\
Race/Ethnicity & 35.9 \\
Non-Hispanic Black (\%) & 35.9 \\
Non-Hispanic White (\%) & 7.7 \\
Non-Hispanic Other (\%) & 20.5 \\
Hispanic ethnicity (\%) & 40 \\
Low SES (\%) & $3.2 \pm 1.8$ \\
Acute severity score ( \pm SD) & $1.0 \pm 1.5$ \\
Charlson Comorbidity Index $( \pm$ SD) & $3.6 \pm 2.3$ \\
ROS (baseline) ( \pm SD) & $6.7 \pm 5.6$ \\
LOS of hospitalized patients ( $( \pm$ SD)
\end{tabular}

$\mathrm{ED}=$ emergency department. LOS = length of stay. Low SES = had marker for low socioeconomic status, either Medicaid insurance or no insurance. 
Table 2. Acute COVID-19 severity variables.

\begin{tabular}{cc}
\hline Severity Variable & $\%$ \\
\hline Intravenous corticosteroid therapy for COVID-19 & 78 \\
Multilobar pneumonia & 73 \\
Peak oxygen requirement $\geq 4 \mathrm{~L} /$ min (or equivalent *) & 70 \\
Remdesivir treatment & 55 \\
Discharged home on supplemental oxygen & 35 \\
Non-invasive ventilation & 10 \\
Decompensated heart failure & 8 \\
Clinical cardiac ischemia & 8 \\
Shock & 3 \\
Endotracheal intubation with mechanical ventilation & 0
\end{tabular}

Column 2 represents percent of the group $(n=40)$ with that variable * Oxygen supplementation 4 LPM or higher or equivalent, such as with a non-rebreather mask.

Table 3. Percentages of patients with abnormal symptom scores.

\begin{tabular}{ccc}
\hline & Survey $\mathbf{1}$ & Survey $\mathbf{2}$ \\
\hline mMRC Dyspnea & 40 & 33 \\
SFQ Fatigue & 53 & 50 \\
PHQ-4 Anxiety & 33 & 18 \\
PHQ-4 Depression & 20 & 10 \\
PHQ-4 Total & 25 & 13 \\
CC8 Cognitive & 18 & 10 \\
\hline
\end{tabular}

No significant changes in any of the outcomes were present.

Table 4. SF-20 health subscale scores.

\begin{tabular}{cccccc}
\hline Subscale & Comparison & First Survey & Second Survey & Change & $\boldsymbol{p .}^{* *}$ \\
\hline Physical Functioning & $74 \pm 23$ & $66 \pm 23$ & $73 \pm 26$ & $7 \pm 19$ & 0.03 \\
Role Functioning & $70 \pm 42$ & $46 \pm 43$ & $61 \pm 46$ & $15 \pm 40$ & 0.02 \\
Social Functioning & $85 \pm 27$ & $49 \pm 42$ & $80 \pm 33$ & $31 \pm 47$ & $<0.01$ \\
Mental Health & $83 \pm 15$ & $69 \pm 21$ & $77 \pm 21$ & $6 \pm 20$ & 0.01 \\
Health Perceptions & $76 \pm 23$ & $46 \pm 30$ & $61 \pm 32$ & $15 \pm 21$ & $< \pm 01$ \\
Pain & $63 \pm 39$ & $62 \pm 28$ & $68 \pm 32$ & $7 \pm 31$ & 0.20 \\
\hline
\end{tabular}

SF-20: Medical Outcomes Study Short Form-20; adjusted scores can range from 0 to 100, with higher scores indicating better health. Change: Mean change from first to second survey. All values are means $\pm \mathrm{SD}$. * Comparison: From a sample of 75 individuals as part of a cross-sectional survey of community-dwelling Canadians not living in institutional settings [12]. This sample was of males and females in the age range 66-70, which is the youngest cohort in this study and closest to our study's mean age. ${ }^{* *}$ Probability of change from first to second survey is $\neq 0$.

Table 5 shows the results of stepwise regression models for each SF-20 subscale in the two surveys. In Survey 1, a high fatigue score was significantly related to health perception and physical and role function. An ROS summary score was related to health perception, social functioning, and mental health. In contrast, baseline high dyspnea was related to all six subscale scores in Survey 2 even when controlling for the baseline score of the corresponding subscale. High fatigue and ROS summary score at baseline were not related to subscale scores in Survey 2. 
Table 5. Factors related to health status in multivariate analysis.

\begin{tabular}{|c|c|c|c|}
\hline SF-20 Subscale & Variable(s) & $p$ & Model $\mathbf{R}^{2} *$ \\
\hline \multicolumn{4}{|l|}{ Survey 1} \\
\hline Physical Function & High fatigue score & $<0.01$ & 0.44 \\
\hline \multirow[t]{2}{*}{ Role Functioning } & High fatigue score & $<0.01$ & 0.44 \\
\hline & Low SES & $<0.01$ & \\
\hline \multirow[t]{2}{*}{ Social Functioning } & ROS summary score & $<0.01$ & 0.30 \\
\hline & Severity score & 0.04 & \\
\hline \multirow[t]{3}{*}{ Mental Health } & ROS summary score & $<0.01$ & \\
\hline & High depression score & $<0.01$ & 0.70 \\
\hline & High anxiety score & 0.02 & \\
\hline \multirow[t]{4}{*}{ Health Perception } & ROS summary score & $<0.01$ & 0.70 \\
\hline & High fatigue score & $<0.01$ & \\
\hline & High severity score & 0.02 & \\
\hline & Charlson Index $\geq 1$ & 0.03 & \\
\hline Pain & Elevated PHQ-4 total score & 0.04 & 0.32 \\
\hline \multicolumn{4}{|l|}{ Survey $2 *$} \\
\hline Physical Function & High dyspnea score $^{1}$ & 0.01 & 0.66 \\
\hline \multirow[t]{2}{*}{ Role Functioning } & High dyspnea score $^{1}$ & 0.01 & 0.51 \\
\hline & Charlson Index > 1 & 0.04 & \\
\hline \multirow[t]{2}{*}{ Social Functioning } & Low SES & 0.01 & 0.28 \\
\hline & High dyspnea score ${ }^{1}$ & 0.01 & \\
\hline \multirow[t]{2}{*}{ Mental Health } & High depression score ${ }^{1}$ & $<0.01$ & 0.52 \\
\hline & High dyspnea score ${ }^{1}$ & $<0.01$ & \\
\hline \multirow[t]{3}{*}{ Health Perception } & High dyspnea score ${ }^{1}$ & $<0.01$ & 0.81 \\
\hline & Charlson Index $>1$ & $<0.01$ & \\
\hline & Low SES & 0.03 & \\
\hline Pain & High dyspnea score $^{1}$ & $<0.01$ & 0.45 \\
\hline
\end{tabular}

* All models controlled for baseline value of corresponding subscale score. ${ }^{1}$ Baseline (Survey 1) score.

Table 6 provides data from the ROS questions. Patients responding "yes" to a question were then asked to state whether that symptom was worse or not worse compared to the time before the COVID-19 infection began. At Survey 1, the most frequently reported symptoms were trouble walking (55\%), cough (50\%), headaches (45\%), arthritis/joint pain $(40 \%)$, and chest pain $(37.5 \%)$. Cough, chest pain, frequent headaches, and loss of or decrease in the sense of smell or taste showed the most improvement from Survey 1 to Survey 2.

Table 6. Review of Systems Variables.

\begin{tabular}{|c|c|c|c|c|}
\hline \multirow[t]{2}{*}{ Variable } & \multicolumn{2}{|c|}{ Survey 1} & \multicolumn{2}{|c|}{ Survey 2} \\
\hline & Present & Worse * & Present & Worse * \\
\hline Cough & 50 & 30 & 18 & 15 \\
\hline Excessive Sleepiness & 33 & 23 & 38 & 23 \\
\hline Chest Pain & 38 & 25 & 18 & 13 \\
\hline Swelling of Ankles or Legs & 25 & 10 & 30 & 18 \\
\hline Frequent Headaches & 45 & 15 & 23 & 18 \\
\hline Numbness of Feet & 18 & 3 & 25 & 8 \\
\hline Arthritis or Joint Pain & 40 & 13 & 48 & 23 \\
\hline Difficulty Walking & 33 & 8 & 33 & 18 \\
\hline Trouble sleeping & 55 & 23 & 45 & 28 \\
\hline Loss or Decrease in Sense of Smell or Taste & 23 & 10 & 8 & 8 \\
\hline
\end{tabular}

Numbers represent percentages of group. Present: present at the time of the interview but not worse compared with pre-COVID-19. * Worse: present and worse compared to time before infection with COVID-19.

\section{Discussion}

A key gap listed in a National Institutes of Health Workshop on Postacute COVID-19 is insufficient knowledge of the natural history of post-acute COVID-19, which would 
require longitudinal analyses [15]. Accordingly, we evaluated the trajectory of health status and common COVID-19 symptoms using two telephone surveys separated by a mean of approximately 57 days. These surveys took place during the second wave of the pandemic in Connecticut in late winter and spring 2021. Patient demographics differ from those hospitalized during the first pandemic wave to hit Connecticut in 2020: those hospitalized in the first wave (that peaked in the spring of 2020) [16] were predominately older Whites, especially nursing home residents. In contrast, our sample was younger, had a relatively high percentage (40\%) with low SES, and had disproportionately high Black and Latino representation. For perspective, 22\% of Connecticut citizens had Medicaid insurance coverage in 2018, with Black and Latino groups comprising $10 \%$ and $16 \%$ of the Connecticut population, respectively $[17,18]$. Racial, ethnic, and socioeconomic discrepancies in hospitalized COVID-19 patients have been noted elsewhere in the United States [19,20].

As expected, based on the existing medical literature on the long-term effects of COVID-19 infection, we demonstrated abnormalities in all the symptom questionnaires out to approximately one and three months. Thus, at the one-month survey, high levels of dyspnea were present in $40 \%$, fatigue in $53 \%$, anxiety in $33 \%$, depression in $20 \%$, and cognitive dysfunction in $18 \%$. Additionally, the review of symptoms analysis at one month was positive in more than $25 \%$ of patients for cough, excessive sleepiness, chest pain, frequent headaches, arthritis/joint pain, difficulty walking, and trouble sleeping. Also noteworthy is that while most of symptom categories showed numerical improvement at approximately three months, none of the questionnaire variables showed statistical improvement. These findings point to the pervasive and long-lasting effects of this disease.

Our primary focus was on long-term effects on heath status, since relatively limited information is published on this important outcome area. Previous investigations have demonstrated persistent abnormalities in other health status instruments, including the respiratory-specific St. George's Respiratory Questionnaire (adapted for COVID-19) [21], the COPD Assessment Test, the EQ-5D-5L, and the Short Form 36 health status questionnaire $[22,23]$. We chose direct administration of the generic measure of health status, the SF-20, to better capture the wide-ranging morbidity from this disease [5]. This instrument, which takes a few minutes to complete and can be administered by telephone survey, has six subscales: physical function, role functioning, social functioning, mental health, health perception, and pain.

Since normative values for the SF-20 are not established and threshold values denoting abnormality for this instrument do not exist, we included for comparison published data from a cohort of male and female Canadians living at home who completed the survey. It is of note that the closest age group in this comparison was of the age range 66-70 years. Despite our patients being considerably younger, the six SF-20 component score means were lower (lower is worse) than in this comparative group, although, of course, statistical comparisons or inferences on minimal clinically-important differences cannot be made. Nonetheless, substantial differences in the subscales of Role Functioning, Social Functioning, Mental Health, and Health Perception are evident, attesting to the long-term impacts of COVID-19 in areas other than on merely physical health. These findings likely reflect added long-lasting psychological and social issues, such as fear, stigma, and social isolation, on the individual.

On multivariable analyses, no single variable stood out in predicting health status components at Survey 1 (fatigue was most prevalent as a predictor, being present in three of six SF-20 components). However higher levels of dyspnea (i.e., mMRC of three or four) was significantly predictive of impairment in all six SF-20 component scores at Survey 2. The reason(s) for these associations are not clear. Our patient sample had severe acute lung disease, as reflected by a 73\% incidence of multilobar pneumonia and the fact that $70 \%$ required high supplemental oxygen at some point during their acute care encounter. It is recognized that Post-COVID-19 Condition may be due in part to long-term lung injury or unresolved inflammation, as suggested by its association with acute disease severity, early dyspnea, and some biomarkers such as D-dimer [24]. It is also possible that other 
symptoms, such as fatigue, might resolve more quickly over time than dyspnea, leaving the latter as a predictor in later follow-up. However, while persistent dyspnea, as a marker for ongoing lower respiratory disease and/or underlying comorbidity, might be expected to explain impairments in health perception or in physical, role, or social functioning, it is difficult to explain the $\mathrm{R}^{2}$ of 0.45 as the sole significant predictor of Pain at Survey 2. An $R^{2}$ of 0.45 indicates that $45 \%$ of the inter-subject variation in pain is explained by an mMRC of 3 or greater. These associations cannot be fully explained by the data on hand. Physiologic data at the time of the surveys might have been helpful, but were not part of this investigation.

While improvement in the symptom questionnaires was minimal and not statistically significant over the mean of 57 days, five of the SF-20 subscales improved significantly over this interval, some approaching those of the (older) comparator group. It is not clear why the observed changes in the generic health status questionnaire were more robust than those of the symptom questionnaires. This may reflect simply differences in individual responsiveness to change among the instruments, although direct comparisons in this measurement property are not available. Alternatively, Social Functioning and Health Perception, which showed the greatest numerical and statistical improvement, may have captured decreasing limitation in physical and social activities and greater self-efficacy perception as the pandemic surge waned and vaccinations became more widespread. Quarantine practices for this disease reduce directly-measured physical activity [25] and adversely affect mental health outcomes [26,27]. Our surveys took place when vaccinations were becoming available to the public. Since vaccination for COVID-19 not only reduces health care utilization and mortality risk, it also appears to reduce long COVID-19 symptoms at 120 days [28], knowledge of vaccination status would be useful in interpreting these results. Unfortunately, our original protocol did not provide for a query on vaccination status, so we cannot evaluate whether vaccinations were a significant factor in these outcomes.

A secondary goal of our study was to evaluate those factors which impact health status. To accomplish this we related demographic factors, socioeconomic status, acute COVID-19 severity, baseline comorbidity, dyspnea, fatigue, psychological variables, cognitive function, and current symptoms (ROS) in multivariate analyses as potential predictors of the health status subscales (Table 5). A number of independent variables entered into the multivariate models predicting health status subscales, attesting to the multidimensional nature of this measurement. However, only high dyspnea entered into every SF-20 subscale model (including Pain) - but only for Survey 2. The reason(s) behind this observation are not clear.

One limitation of this study and other COVID-19 observational studies evaluating persistent effects of this disease is that pre-infection, baseline data are not available for comparison. For instance, we demonstrated elevated dyspnea to be present in $40 \%$ at approximately one month after health care discharge. What portion of this $40 \%$ is new and secondary to COVID-19 and what portion is not new but rather due to pre-existing disease? Adding to this dilemma is the fact that COVID-19 infection is more common in those with a pre-existing disease burden and therefore more likely to have chronic symptoms.

Another limitation of our study is that we surveyed only a small number of patients in one health care system over a relatively short period of time and with discharge to a home setting only. Only about one-half of the patients eligible for inclusion in the study were able to be contacted by telephone, and of those who completed the first survey, $26 \%$ did not participate in the second. Indeed, dropouts were younger and had a lower acute severity score, creating problems with interpretation of the data. Our surveys coincided with the tail end of the second COVID-19 surge that peaked in Connecticut in mid-January, 2021 [29]; in contrast to the first surge, it tended to produce clinical disease in younger individuals. Adding to this limitation of generalizability was the availability of COVID-19 vaccines (which were not analyzed in our study) over the span of the surveys. Although the number receiving vaccines was probably low as our study commenced, uptake was probably higher by the second survey. While vaccination for this disease reduces both infectivity rate and 
clinical severity of the infection, limited data suggest that full vaccination may also reduce symptoms out to 28 days or longer in those with breakthrough infections [30], possibly influencing our results.

As mentioned earlier, since our severity score was not validated, inferences on acute severity (not) predicting the persistence of symptoms must be made with caution. Additionally, the comorbid conditions making up the Charlson Comorbidity Index may not be relevant in predicting sequelae to COVID-19 infection, although another version of the Charlson Index did demonstrate that a score above 0 was related to an increased risk of severe COVID-19 and death [14]. It is of note that none of the other potentially relevant coexisting conditions that we included-obesity, hypertension, and asthma-predicted long-term impairment in health status.

In summary, our findings are similar to other published observational studies in that we demonstrated the long-term effects of COVID-19 infection across multiple symptom PRO areas and health status. Our data differ somewhat in that we measured outcomes at two times, approximately 57 days apart, giving insight into longitudinal changes in post-acute COVID-19. Health status, which may capture a wider aspect of impact from the disease and its sequelae, seemed to show more improvement over this interval. The implication from our study is that clinicians should be cognizant of the long-term effects of the disease in some individuals in order to initiate therapy, when indicated.

In conclusion, we demonstrated substantial impairments in multiple aspects of general health status out to one month and, to lesser degree, out to three months. Persistent dyspnea appears to be a factor in prolonged health status limitation. Taking this into consideration, comprehensive pulmonary rehabilitation may be of benefit for those with ongoing health status impairment. This intervention, which includes exercise training, promotion of self-efficacy through collaborative self-management, and care coordination exerts its often-substantial beneficial effects though ameliorating the effects of systemic or comorbid illness [31,32]. Recognizing the long-term symptoms and functional limitation of some COVID-19 patients, the University of Leicester has recently started a rehabilitation program for these patients [33]. Further investigation of its effectiveness in this particular situation needs to be determined.

Author Contributions: Conceptualization, R.Z., A.K.; methodology: R.Z., A.K., D.W.; validation, D.W., formal analysis, R.Z., D.W., investigation, R.Z., C.M., S.C. (Suzanne Carpe), S.C. (Soontharee Congrete), H.S., J.R.; resources, C.S.; data curation, R.Z., D.W.; writing-original draft preparation: C.M., S.C. (Suzanne Carpe), R.Z.; writing-review and editing, all authors; supervision, C.S., R.Z. All authors have read and agreed to the published version of the manuscript.

Funding: This research received no external funding.

Institutional Review Board Statement: This study was approved by our Institutional Review Board (Trinity Health of New England Institutional Review Board (IRB), Assurance \#FWA00020300, approval 28 September 2021).

Informed Consent Statement: Informed consent was obtained via telephone, per IRB direction.

Data Availability Statement: Interview and medical record data supporting the results and conclusions of this study were generated at St Francis Hospital, Hartford, CT. De-identified data supporting the findings of this study may be available via written request to the corresponding author.

Conflicts of Interest: The authors declare no conflict of interest.

\section{References}

1. Goërtz, Y.M.J.; Van Herck, M.; Delbressine, J.M.; Vaes, A.W.; Meys, R.; Machado, F.V.C.; Houben-Wilke, S.; Burtin, C.; Posthuma, R.; Franssen, F.M.E.; et al. Persistent symptoms 3 months after a SARS-CoV-2 infection: The post-COVID-19 syndrome? ERJ Open Res. 2020, 6, 00542-02020. [CrossRef] [PubMed]

2. Logue, J.K.; Franko, N.M.; McCulloch, D.J.; McDonald, D.; Magedson, A.; Wolf, C.R.; Chu, H.Y. Sequelae in Adults at 6 Months After COVID-19 Infection. JAMA Netw. Open 2021, 4, e210830. [CrossRef] [PubMed]

3. Lopez-Leon, S.; Wegman-Ostrosky, T.; Perelman, C.; Sepulveda, R.; Rebolledo, P.A.; Cuapio, A.; Villapol, S. More than 50 long-term effects of COVID-19: A systematic review and meta-analysis. Sci. Rep. 2021, 11, 1-12. [CrossRef] [PubMed] 
4. Soriano, J.B.; Murthy, S.; Marshall, J.C.; Relan, P.; Diaz, J.V. Clinical Case Definition Working Group on Post-COVID-19 Condition. A Clinical Case Definition of Post-COVID-19 Condition by a Delphi Consensus; WHO: Geneva, Switzerland, 2021.

5. Guyatt, G.H. A taxonomy of health status instruments. J. Rheumatol. 1995, 22, 1188-1190.

6. Guyatt, G.H. Measurement of health-related quality of life in heart failure. J. Am. Coll. Cardiol. 1993, 22, 185A-191A. [CrossRef]

7. Mahler, D.A.; Wells, C.K. Evaluation of clinical methods for rating dyspnea. Chest 1988, 93, 580-586. [CrossRef] [PubMed]

8. Kroenke, K.; Spitzer, R.L.; Williams, J.B.; Lowe, B. An ultra-brief screening scale for anxiety and depression: The PHQ-4. Psychosomatics 2009, 50, 613-621.

9. Damin, A.E.; Nitrini, R.; Brucki, S.M.D. Cognitive Change Questionnaire as a method for cognitive impairment screening. Dement Neuropsychol. 2015, 9, 237-244. [CrossRef]

10. Penson, A.; van Deuren, S.; Worm-Smeitink, M.; Bronkhorst, E.; Hoogen, F.H.V.D.; van Engelen, B.G.; Peters, M.; Bleijenberg, G.; Vercoulen, J.H.; Blijlevens, N.; et al. Short fatigue questionnaire: Screening for severe fatigue. J. Psychosom. Res. 2020, 137, 110229. [CrossRef]

11. Stewart, A.L.; Hays, R.D.; Ware, J.E., Jr. The MOS short-form general health survey. Reliability and validity in a patient population. Med. Care 1988, 26, 724-735. [CrossRef]

12. Carver, D.J.; Chapman, C.A.; Thomas, V.S.; Stadnyk, K.J.; Rockwood, K. Validity and reliability of the Medical Outcomes Study Short Form-20 questionnaire as a measure of quality of life in elderly people living at home. Age Ageing 1999, 28, 169-174. [CrossRef]

13. Quan, H.; Li, B.; Couris, C.M.; Fushimi, K.; Graham, P.; Hider, P.; Januel, J.-M.; Sundararajan, V. Updating and Validating the Charlson Comorbidity Index and Score for Risk Adjustment in Hospital Discharge Abstracts Using Data From 6 Countries. Am. J. Epidemiol. 2011, 173, 676-682. [CrossRef] [PubMed]

14. Christensen, D.M.; Strange, J.E.; Gislason, G.; Torp-Pedersen, C.; Gerds, T.; Fosbøl, E.; Phelps, M.D. Charlson Comorbidity Index Score and Risk of Severe Outcome and Death in Danish COVID-19 Patients. J. Gen. Intern. Med. 2020, 35, 2801-2803. [CrossRef]

15. Lerner, A.M.; Robinson, D.A.; Yang, L.; Williams, C.F.; Newman, L.M.; Breen, J.J.; Eisinger, R.W.; Schneider, J.S.; Adimora, A.A.; Erbelding, E.J. Toward Understanding COVID-19 Recovery: National Institutes of Health Workshop on Postacute COVID-19. Ann. Intern. Med. 2021, 174, 999-1003. [CrossRef]

16. Center JHUCR. 2021. Available online: https:// coronavirus.jhu.edu/region/us/connecticut (accessed on 28 September 2021).

17. Bureau USC. QuickFacts, Hartford County, Connecticut. 2019. Available online: https://www.census.gov/quickfacts/fact/table/ hartfordcountyconnecticut/PST045219 (accessed on 29 October 2021).

18. Connecticut COVID-19 Case Tracking. 202. Available online: https:/ / public.tableau.com/app/profile/connecticut.state.data. center/viz/ConnecticutCOVID-19CaseTracking/CTdataCollaborativeCOVID-19 (accessed on 28 September 2021).

19. Karaca-Mandic, P.; Georgiou, A.; Sen, S. Assessment of COVID-19 Hospitalizations by Race/Ethnicity in 12 States. JAMA Intern Med. 2021, 181, 131-134. [CrossRef] [PubMed]

20. Hsu, H.E.; Ashe, E.M.; Silverstein, M.; Hofman, M.; Lange, S.J.; Razzaghi, H.; Mishuris, R.G.; Davidoff, R.; Parker, E.M.; PenmanAguilar, A.; et al. Race/Ethnicity, Underlying Medical Conditions, Homelessness, and Hospitalization Status of Adult Patients with COVID-19 at an Urban Safety-Net Medical Center-Boston, Massachusetts, 2020. MMWR. Morb. Mortal. Wkly. Rep. 2020, 69, 864-869. [CrossRef]

21. Jones, R.; Davis, A.; Stanley, B.; Julious, S.; Ryan, D.; Jackson, D.J.; Halpin, D.M.; Hickman, K.; Pinnock, H.; Quint, J.K.; et al. Risk Predictors and Symptom Features of Long COVID Within a Broad Primary Care Patient Population Including Both Tested and Untested Patients. Pragmatic Obs. Res. 2021, 12, 93-104. [CrossRef]

22. Chen, K.Y.; Li, T.; Gong, F.H.; Zhang, J.S.; Li, X.K. Predictors of Health-Related Quality of Life and Influencing Factors for COVID-19 Patients, a Follow-Up at One Month. Front. Psychiatry 2020, 11, 668. [CrossRef]

23. Van der Sar-van der Brugge, S.; Talman, S.; Winter, L.B.-D.; de Mol, M.; Hoefman, E.; van Etten, R.W.; De Backer, I.C. Pulmonary function and health-related quality of life after COVID-19 pneumonia. Respir. Med. 2021, 176, 106272. [CrossRef] [PubMed]

24. Yong, S.J. Long-Haul COVID-19: Putative Pathophysiology, Risk Factors, and Treatments. Preprints. 2020. Available online: https:/ / www.preprints.org/manuscript/202012.0242/v1 (accessed on 7 January 2022).

25. López-Sánchez, G.F.; López-Bueno, R.; Gil-Salmerón, A.; Zauder, R.; Skalska, M.; Jastrzębska, J.; Jastrzębski, Z.; Schuch, F.B.; Grabovac, I.; Tully, M.A.; et al. Comparison of physical activity levels in Spanish adults with chronic conditions before and during COVID-19 quarantine. Eur. J. Public Health 2021, 31, 161-166. [CrossRef]

26. Wang, Y.; Shi, L.; Que, J.; Lu, Q.; Liu, L.; Lu, Z.; Xu, Y.; Liu, J.; Sun, Y.; Meng, S.; et al. The impact of quarantine on mental health status among general population in China during the COVID-19 pandemic. Mol. Psychiatry 2021, 26, 4813-4822. [CrossRef] [PubMed]

27. Mechili, E.A.; Saliaj, A.; Kamberi, F.; Girvalaki, C.; Peto, E.; Patelarou, A.E.; Bucaj, J.; Patelarou, E. Is the mental health of young students and their family members affected during the quarantine period? Evidence from the COVID-19 pandemic in Albania. $J$. Psychiatr. Ment. Health Nurs. 2021, 28, 317-325. [CrossRef] [PubMed]

28. Tran, V.T.; Perrodeau, E.; Saldanha, J.; Pane, I.; Ravaud, P. Efficacy of COVID-19 vaccination on the symptoms of patients with long COVID: A target trial emulation using data from the ComPaRe e-Cohort in France. Lancet, 2021; Preprint. Posted 29 September. [CrossRef]

29. Worldometer. Daily New Deaths in Connecticut. 2021. Available online: https://www.worldometers.info/coronavirus/usa/ connecticut/ (accessed on 27 August 2021). 
30. Antonelli, M.; Penfold, R.S.; Merino, J.; Sudre, C.H.; Molteni, E.; Berry, S.; Canas, L.S.; Graham, M.S.; Klaser, K.; Modat, M.; et al. Risk factors and disease profile of post-vaccination SARS-CoV-2 infection in UK users of the COVID Symptom Study app: A prospective, community-based, nested, case-control study. Lancet Infect. Dis. 2021, 22, 43-55. [CrossRef]

31. Nici, L.; ZuWallack, R.; Wouters, E.; Donner, C.F. On pulmonary rehabilitation and the flight of the bumblebee: The ATS/ERS Statement on Pulmonary Rehabilitation. Eur. Respir. J. 2006, 28, 461-462. [CrossRef]

32. Nici, L.; ZuWallack, R. Integrated Care in Chronic Obstructive Pulmonary Disease and Rehabilitation. COPD 2018, 15, 223-230. [CrossRef]

33. Daynes, E.; Gerlis, C.; Singh, S.J. The demand for rehabilitation following COVID-19: A call to service providers. Physiotherapy 2021, 113, A1-A3. [CrossRef] 